\title{
Intermediate phase of the one dimensional half-filled Hubbard-Holstein model
}

\author{
R.T. Clay and R.P. Hardikar ${ }^{1}$ \\ ${ }^{1}$ Department of Physics and Astronomy and ERC Center for Computational Sciences, \\ Mississippi State University, Mississippi State MS 39762
}

(Dated: June 17, 2021)

\begin{abstract}
We present a detailed numerical study of the Hubbard-Holstein model in one dimension at half filling, including full finite-frequency quantum phonons. At half filling, the effects of the electronphonon and electron-electron interactions compete, with the Holstein phonon coupling acting as an effective negative Hubbard onsite interaction $U$ that promotes on-site electron pairs and a Peierls charge-density wave state. Most previous work on this model has assumed that only Peierls or $U>0$ Mott insulator phases are possible at half filling. However, there has been speculation that a third metallic phase exists between the Peierls and Mott phases. We present results confirming the intermediate metallic phase, and show that the Luttinger liquid correlation exponent $K_{\rho}>1$ in this region, indicating dominant superconducting pair correlations. We explore the full phase diagram as a function of onsite Hubbard $U$, phonon coupling constant, and phonon frequency.

PACS numbers: 71.10.Fd, 71.30.+h, 71.45.Lr
\end{abstract}

Electron-phonon (e-ph) interactions can give rise to a number of interesting effects in low-dimensional materials, including superconductivity as well as charge-density wave and insulating phenomena. Frequently these materials feature strong electron-electron (e-e) interactions as well, leading to very rich phase diagrams that combine lattice, charge, and spin (magnetic) orderings. We will focus specifically on materials where the electrons are coupled to localized vibrational modes, which may be of relatively high frequency. This type of e-ph interaction is most studied in molecular crystal materials, including the quasi-one- and quasi-two-dimensional organic superconductors 1 and fullerene superconductors 2]. In all of these materials, a fundamental question is whether the effects of e-e and e-ph interactions compete or cooperate with each other. In this Letter, we examine this issue within one of the most basic models. We find that despite the two interactions each separately favoring insulating states, together they can mediate an unexpected metallic phase with superconducting (SC) pair correlations.

The model we consider is the one-dimensional (1D) Hubbard-Holstein model (HHM), with Hamiltonian

$$
\begin{aligned}
H & =-t \sum_{j, \sigma}\left(c_{j+1, \sigma}^{\dagger} c_{j, \sigma}+\text { h.c. }\right)+U \sum_{j} n_{j, \uparrow} n_{j, \downarrow} \\
& +g \sum_{j, \sigma}\left(b_{j}^{\dagger}+b_{j}\right) n_{j, \sigma}+\omega \sum_{j} b_{j}^{\dagger} b_{j},
\end{aligned}
$$

where $c_{j, \sigma}^{\dagger}\left(c_{j, \sigma}\right)$ are fermionic creation (annihilation) operators for electrons on site $j$ with spin $\sigma, b_{j}^{\dagger}\left(b_{j}\right)$ are bosonic creation (annihilation) operators for phonons at site $j$, and $n_{j, \sigma}=c_{j, \sigma}^{\dagger} c_{j, \sigma}$. The dispersionless phonons have frequency $\omega$ and are coupled to the local electron density with coupling strength $g[3]$. $U$ is the Hubbard on-site e-e interaction energy. All energies will be given below in units of the hopping integral $t$.
The properties of the 1D 1/2-filled HHM are well understood in two limits: the static or $\omega \rightarrow 0$ and the $\omega \rightarrow \infty$ limit [4]. First, in the static limit, the ground state is Peierls distorted for any nonzero e-ph coupling $g=0^{+}$. As the phonons couple to the electron density, the Peierls state is a $2 \mathrm{k}_{F}$ charge-density wave (CDW) consisting of alternating large and small site charges. In the $\omega \rightarrow \infty$ limit, the retarded interaction between electrons mediated by the phonons becomes instantaneous in imaginary time, and the phonons may be integrated out. This leads to an effective renormalized Hubbard interaction $U_{\text {eff }}=U-2 g^{2} / \omega$. While strictly at $\omega \rightarrow \infty$ the Peierls state cannot occur, for finite $\omega$ the Peierls state may again occur, although the mapping to an effective negative $U$ is no longer exact. However, based on the $\omega \rightarrow \infty$ mapping, it has been believed that the ground state phase of the HHM may be determined through $U_{\text {eff. }}$. $U_{\text {eff }}$ should correspond to the $U>0$ Hubbard model, which in $1 \mathrm{D}$ at $1 / 2$ filling has a finite charge gap for any $U>0$ and no spin gap. We shall refer to this state as the Mott state. On the other hand, if $U_{\text {eff }}<0$, e-ph interactions dominate over e-e interactions, and the ground state is Peierls CDW distorted.

Hence at $\omega=0$, the Peierls distortion occurs unconditionally, while at $\omega=\infty$ there is no Peierls state. Much less is known in the intermediate $\omega$ region. In the $U=0$ model, the Peierls state may be viewed as a traditional band insulator for small $\omega$, and as bipolaronic insulator composed of tightly bound pairs in the large $\omega$ limit, with a crossover between these pictures for $\omega \sim t[5]$. If $\omega>0$, the Peierls distortion only occurs for e-ph coupling $g$ above some critical value $g_{c}, \underline{6}$, , 7]. For $g<g_{c}$, the ground state is then metallic at $1 / 2$ filling (at $U=0$ ). For $U=0, g_{c}$ has been calculated using a functional integral method [6] and also via the density matrix renormalization group (DMRG) 7]. For intermediate $U$ as well as $\omega$, far less is known. However, it has been recently proposed 


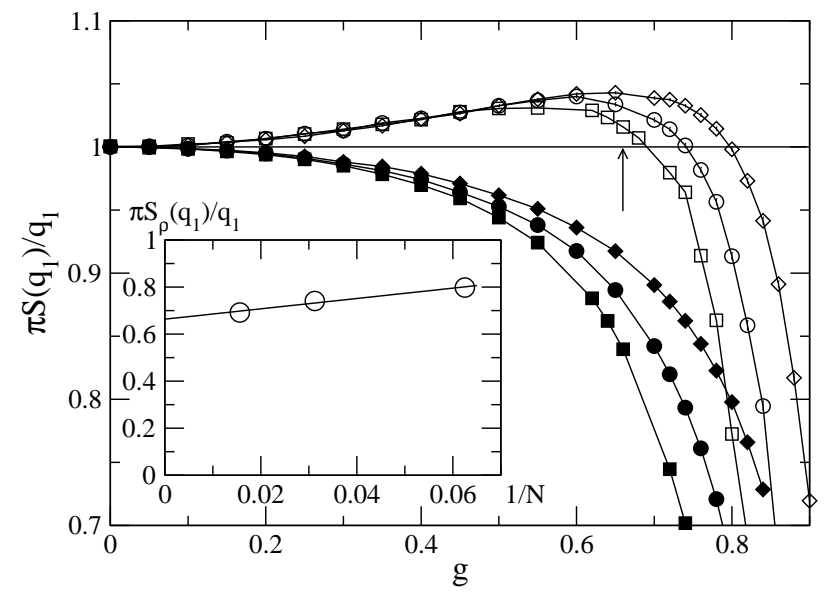

FIG. 1: $U=0, \omega=1$ results for long-wavelength charge (open symbols) and spin (filled symbols) structure factors versus $g$ for periodic systems of $N=16$ (diamonds), $N=32$ (circles), and $N=64$ (squares) sites. Statistical errors are smaller than the symbols. The inset shows finite-size scaling of the critical coupling $g_{c}$ (indicated by arrow) where $K_{\rho}=1$.

that a metallic ground state exists intermediate between the Mott and Peierls states at $1 / 2$ filling, i.e. when $U_{\text {eff }}$ is close to zero 8]. This metallic phase occurs for intermediate $\omega$ and hence cannot be predicted from the small and large $\omega$ limits. Our goal in this Letter is to confirm this metallic state and investigate its properties. Its existence is perhaps not surprising given the known existence of a metallic phase for $g<g_{c}$ at $U=0$; this region of the phase diagram continues to exist for finite $U$. We further show that this metallic region persists for a substantial range of parameters provided $\omega$ is not too small.

Hamiltonian Eq. 1 is difficult to analyze in the intermediate coupling region due to the presence of both electrons and phonons. The numerical method we use is the Stochastic Series Expansion (SSE) quantum Monte Carlo method with directed loops 9]. SSE is a powerful method for non-frustrated quantum spin systems or 1D electron lattice models where no sign problem occurs. Importantly, there are no approximations in the method besides finite system size and temperature. Electron-phonon interactions have been incorporated in SSE for both spin models [10] and electron models 11]. As in these references, we treat the phonons in the occupation-number basis. The number of phonons per lattice site is unbounded in the thermodynamic limit, but for a finite system at a finite temperature, the number of phonons may be truncated. We choose this truncation in a similar manner as the truncation of sequence length in the SSE method [9]: in the equilibration phase of the calculation, the phonon truncation is increased to exceed the current number of phonons on any given lattice site whenever necessary. All results shown below used periodic lattices of $N$ sites, with inverse temperatures of at least $\beta / t=2 N$ and phonon cutoffs of up to 30 phonons per lat- tice site. In this Letter we focus on results for the HHM model, and details on the SSE implementation will be published separately. Our code was checked extensively against Lanczös exact diagonalization results for several different observables. We also implemented the quantum parallel tempering algorithm 12], where different processors of a parallel computer are assigned different model parameters $(U, g$, and $\omega)$. A Metropolis probability is then computed to switch configurations between processors with adjacent parameters. As in reference 12, we found this technique essential in obtaining smooth data across quantum phase transition boundaries.

The low energy properties of any 1D gapless interacting electron model may be mapped to an effective continuum model, or Luttinger Liquid (LL). The properties of the LL, and in particular the decay with distance of different correlation functions are then described by two correlation exponents, $K_{\rho}$ for charge properties, and $K_{\sigma}$ for spin [13]. $K_{\rho}$ values greater than 1 indicate dominant attractive SC correlations (no SC long-range order is possible in strictly $1 \mathrm{D}$ systems), while $K_{\rho}<1$ corresponds to repulsive charge correlations. For models with spin-rotation symmetry, $K_{\sigma}$ is always equal to $1 . K_{\sigma}=0$ then indicates the presence of a spin gap. These exponents are most easily computed via the SSE data from the static structure factors:

$$
S_{\rho, \sigma}(q)=\frac{1}{N} \sum_{j, k} e^{i q(j-k)}\left\langle\left(n_{j \uparrow} \pm n_{j \downarrow}\right)\left(n_{k \uparrow} \pm n_{k \downarrow}\right)\right\rangle
$$

$K_{\rho}$ and $K_{\sigma}$ are then proportional to the slope of the corresponding structure factor in the long wavelength limit $q \rightarrow 0$ [14]:

$$
K_{\rho, \sigma}=\frac{1}{\pi q} S_{\rho, \sigma}(q \rightarrow 0)
$$

These values once finite-size scaled may then be used to determine the quantum phase boundaries. A second observable we will use are the charge and spin stiffnesses, $\rho_{c}$ and $\rho_{s}$, measured in the SSE method via the winding number [12]. A zero stiffness indicates a gap in the corresponding sector, while nonzero $\rho$ indicates no gap. We also verified directly that charge-charge (spin-spin) correlation functions showed staggered order in the Peierls (Mott) phases.

We first present results for $U=0$ and $\omega=1$. Fig. 11 shows the slope of charge and spin structure factors $\pi S_{\rho, \sigma}\left(q_{1}\right) / q_{1}$ evaluated at the smallest wavevector $q_{1}=2 \pi / N$, plotted versus e-ph coupling $g$. For $g \rightarrow 0$ both $K_{\rho}$ and $K_{\sigma}$ tend to exactly 1 , as required for free electrons. Finite size effects become very small in this limit, consistent with the expected vanishing of logarithmic corrections when $K_{\rho}=K_{\sigma}=1$ [11, 15]. At a critical coupling $g_{c}, K_{\rho}$ crosses 1 and tends to zero, marking the transition to the Peierls state. From finite-size scaling of 16,32 , and 64 site systems vs. $1 / N$ (see Fig. 1] inset), we find the critical coupling $g_{c}=0.66 \pm 0.01$, with 

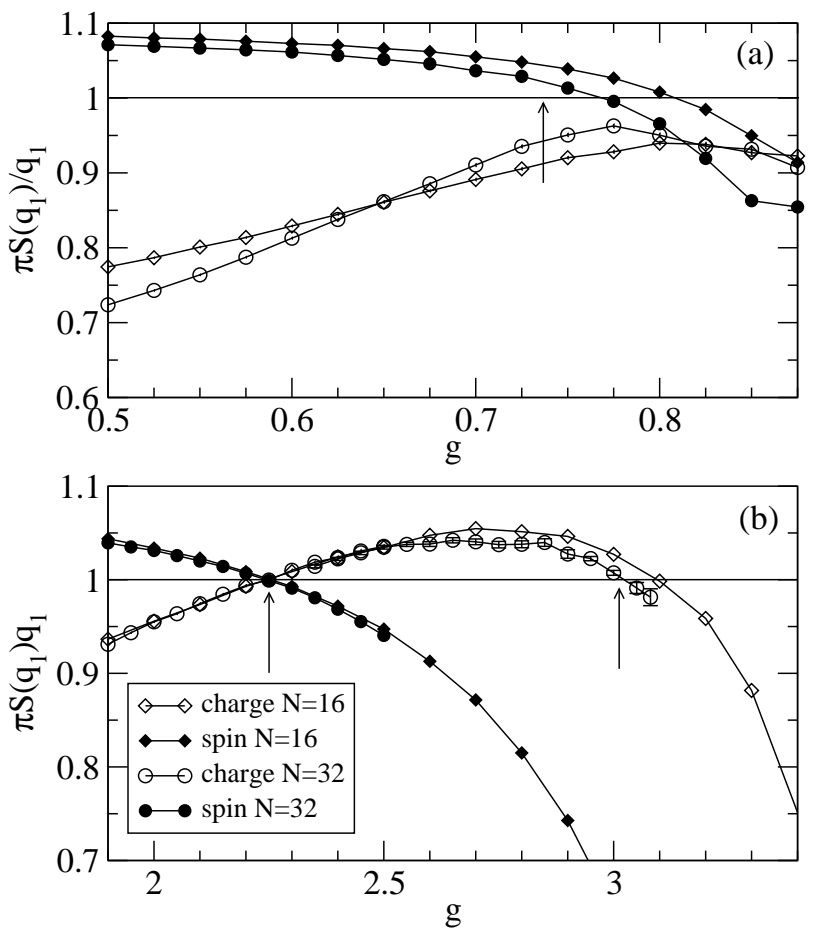

FIG. 2: Long-wavelength charge (open symbols) and spin (filled symbols) structure factors versus $g$. (a) $U=2, \omega=0.5$; arrow indicates finite-size scaled $g_{c}$ (b) $U=2, \omega=5$; arrows indicate finite-size scaled transitions $g_{c 1}$ and $g_{c 2}$.

the uncertainty estimated from the linear fit. A previous DMRG study, which did not attempt finite-size scaling found $g_{c} \approx 0.8$ [7]. For $g<g_{c}$, the LL exponent $K_{\rho}>1$, and $K_{\sigma}$ scales to zero with increasing $N$. Therefore, for $U=0$ and $g<g_{c}$, the ground state is metallic, with dominant SC pair correlations and a spin gap.

For any finite $U$ at $g=0$ and $1 / 2$ filling, the dominant ground state correlations of Eq. प are spin-density wave (SDW). In this ground state $K_{\sigma}=1$, and $K_{\rho}=0$ indicating an insulating state with no spin gap. As this Mott state is not equivalent to the metallic state found for $g<g_{c}$ in Fig. 10 we may then expect the possibility of three different phases for the HHM with $U>0$ and $g>0$ : Mott, Peierls, and metallic SC. Fig. 2 shows the charge and spin structure factors again versus $g$ with $U=2$. We first discuss results for small phonon frequency $\omega=0.5$ shown in Fig. 2(a). We find that for small $g, K_{\sigma}$ tends toward 1 as system size increases. At a critical coupling $g_{c}, K_{\sigma}$ becomes less than 1, indicating the opening of a spin gap and the transition to the Peierls state. The charge exponent $K_{\rho}$ scales towards zero on both sides of the transition, developing a peak at $g=g_{c}$. Similar results are found for the $1 / 2$-filled $1 \mathrm{D}$ extended Hubbard model (EHM), where $K_{\rho}$ also peaks at the transition between CDW and bond-order wave (BOW) phases 16. The finite value of $K_{\rho}$ on the boundary indicates that the transition is of continuous nature [16]. Like the CDW-

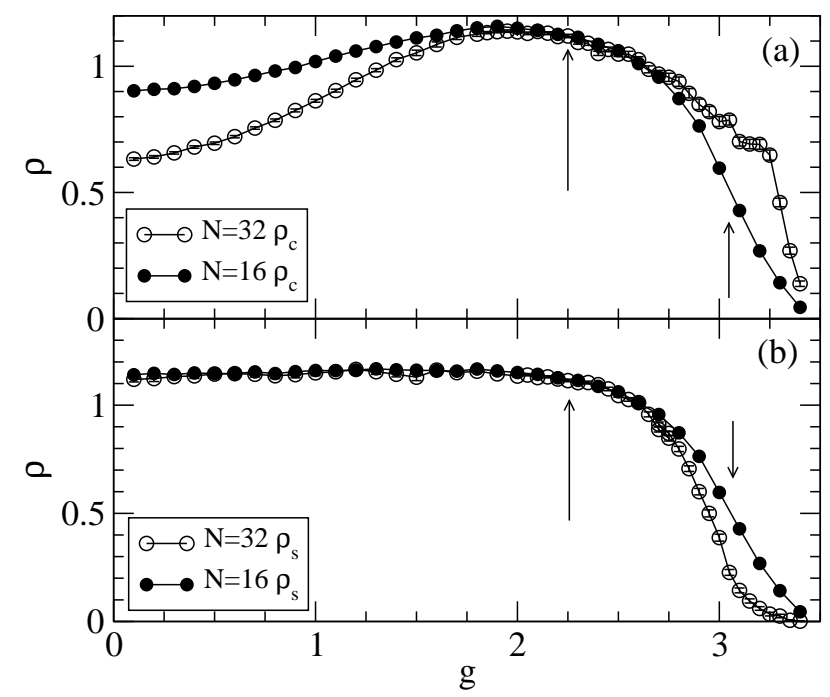

FIG. 3: Charge (a) and spin (b) stiffnesses versus $g$ for $U=2$, $\omega=5$. Open (closed) symbols are for $N=32$ (16) site lattices. Arrows mark the transition points $g_{c 1}$ and $g_{c 2}$ determined from Fig. 2(b).

(BOW)-SDW transition in the 1/2-filled EHM, we find similar behavior for small $\omega$ in the HHM as $U$ increases: the value of $K_{\rho}$ at the transition decreases with $U$, indicating that for large $U$ the transition is first order rather than continuous. The correspondence is not surprising, as the effect of a nearest-neighbor interaction $V \gg U$ can be viewed as an effective negative $U[14$.

Charge and spin response typical for large $\omega$ are shown in Fig. 2(b), here shown for $U=2$ and $\omega=5$. Again, for small $g$, we find $K_{\sigma}$ tends toward 1 as system size increases, and $K_{\rho}$ tends toward 0, consistent with the Mott state. However, at a critical coupling $g_{c 1} K_{\rho}$ exceeds 1 and $K_{\sigma}$ becomes less than one, indicating the dominant-SC state found for $U=0, g<g_{c}$. The value of $g_{c 1}$ is very close to the expected value where $U_{\text {eff }}=0$. Increasing $g$ further, for $g=g_{c 2} K_{\rho}$ becomes less than 1 , indicating the opening of a charge gap and the Peierls state. In the intermediate region $g_{c 1}<g<g_{c 2}$, the properties of the model are identical to the $U=0$ model for $g<g_{c}$ as seen in Fig. [1 i.e. metallic with dominant $\mathrm{SC}$ correlations. While the second transition point $g_{c 2}$ is finite-size dependent, finite-size effects are very weak at the first transition since $K_{\rho}=K_{\sigma}=1$. It is then clear that due to the crossing of $K_{\rho}$ and $K_{\sigma}$ curves at exactly 1 at $g=g_{c 1}$, a region of $K_{\rho}>1$ must exist for $g>g_{c 1}$.

To confirm the structure factor results indicating two transitions at $g_{c 1}$ and $g_{c 2}$, we present the charge $\left(\rho_{c}\right)$ and spin $\left(\rho_{s}\right)$ stiffnesses in Fig. 3. As with the structure factors, the presence of two transitions may only be detected from $\rho_{c}$ and $\rho_{s}$ after finite-size scaling has been performed. For clarity, we plot only two system sizes in Fig. [3] In Fig. 3(a) we see that for small $g, \rho_{c}$ decreases with system size, indicating a charge gap. $\rho_{s}$ is nearly 


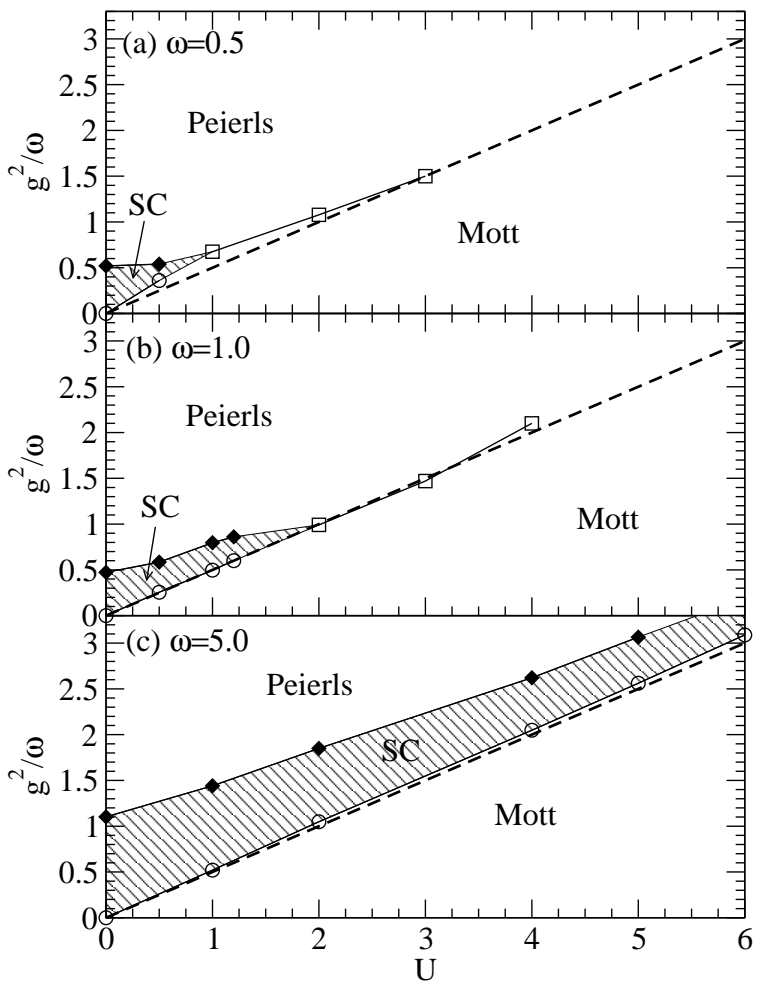

FIG. 4: Evolution of the intermediate phase versus $\omega$. (a) $\omega=0.5$ (b) $\omega=1$ (c) $\omega=5$. Open circles indicate the boundary $g_{c 1}$ between Mott and SC phases, filled diamonds the boundary $g_{c 2}$ between SC and Peierls phases, and open squares the boundary between Mott and Peierls phases. Dashed line indicates $U=2 g^{2} / \omega$.

constant with system size, indicating no spin gap. In the intermediate phase, this is reversed, with $\rho_{c}$ remaining constant or increasing with system size, and $\rho_{s}$ decreasing with system size. This again indicates a spin gap but no charge gap in the SC region. Finally, for $g>g_{c 2}$, both stiffnesses go to zero in the Peierls state.

Fig. 4 shows the evolution of the intermediate phase as a function of $\omega$, with phase boundaries determined from finite-size scaling of 16,24 , and 32 site structure factor data. In all cases, the SC phase exists for $g<g_{c}$ exactly at $U=0$. As $U$ is increased at fixed $\omega$, the $\mathrm{SC}$ region then shrinks. For small enough $\omega$, we see two different sequences of phases, either Mott-SC-Peierls for small $U$ (as in Fig. 2(b)), or Mott-Peierls (as in Fig. 2(a)) for large $U$. Numerically it is difficult to precisely determine point where all three phases meet, but we find that the SC phase disappears at $U \approx 1$ for $\omega=0.5$, and at $U \approx 2$ for $\omega=1.0$. In Fig. 4(a) and (b), we have not plotted points for the Mott/Peierls boundary for large $U$, as in this region the transition becomes strongly first order, making exact determination of the boundary difficult. However, the transition appears to remain close to $g^{2} / \omega=U / 2$. For $\omega=5$, we were not able to access large enough $U$ to determine the upper cutoff $U$ neces- sary to suppress the $\mathrm{SC}$ state, but the intermediate phase appears to persist up to at least $U \approx 7$ for $\omega=5$. In all cases, we find the line $U_{\text {eff }}=0$ to very accurately predict either the Mott-SC or Mott-Peierls boundary, but not the SC-Peierls boundary. Our phase diagram is slightly different from reference 8 , where the metallic region was found to extend to either side of the $U_{\text {eff }}=0$ line.

In conclusion, we have shown that a metallic SC region exists intermediate between Peierls and Mott phases in the 1D 1/2-filled HHM. While in the $1 / 2$-filled model with small phonon frequency $\omega<t$ the intermediate phase occupies a relatively small region of the phase diagram, we expect that in the presence of doping (in fact most of the organic SC's are 1/4-filled [1]), the size of this region will be greatly enhanced. Furthermore, while the SC pairing found here exactly at 1/2-filling consists of on-site pairs or bipolarons, with doping both onsite and nearest-neighbor pairing may be mediated by the Holstein phonons 17. We are currently exploring these possibilities in the doped model.

This work was supported by Oak Ridge Associated Universities. We thank A. Sandvik and P. Sengupta for discussions regarding the SSE method. Numerical calculations were performed at the Mississippi State University ERC Center for Computational Sciences.

[1] T. Ishiguro, K. Yamaji, and G. Saito, Organic Superconductors (Springer-Verlag, New York, 1998).

[2] O. Gunnarsson, Rev. Mod. Phys. 69, 575 (1997).

[3] T. Holstein, Ann. Phys. 8, 325 (1959).

[4] J. E. Hirsch and E. Fradkin, Phys. Rev. B 27, 1680 (1983).

[5] H. Fehske, G. Wellein, G. Hager, A. Wei $\beta$ e, and A. R. Bishop, Phys. Rev. B 69, 165115 (2004).

[6] C. Wu, Q. Huang, and X. Sun, Phys. Rev. B 52, R15683 (1995).

[7] E. Jeckelmann, C. Zhang, and S. White, Phys. Rev. B 60, 7950 (1999).

[8] Y. Takada and A. Chatterjee, Phys. Rev. B 67, 081102R (2003).

[9] O. F. Syljuasen and A. W. Sandvik, Phys. Rev. E 66, 046701 (2002).

[10] A. W. Sandvik and D. K. Campbell, Phys. Rev. Lett. 83, 195 (1999).

[11] P. Sengupta, A. W. Sandvik, and D. K. Campbell, Phys. Rev. B 67, 245103 (2003).

[12] P. Sengupta, A. W. Sandvik, and D. K. Campbell, Phys. Rev. B 65, 155113 (2002).

[13] J. Voit, Rep. Prog. Phys. 58, 977 (1995).

[14] R. T. Clay, A. W. Sandvik, and D. K. Campbell, Phys. Rev. B 59, 4665 (1999).

[15] S. Eggert, Phys. Rev. B 54, R9612 (1996).

[16] A. W. Sandvik, L. Balents, and D. K. Campbell, Phys. Rev. Lett. 92, 236401 (2004).

[17] J. Bonča, T. Katras̆nik, and S. A. Trugman, Phys. Rev. Lett. 84, 3153 (2000). 